\title{
La integración curricular como experiencia de aprendizaje: Hoja de ruta para su aplicación en dos cursos de formación docente en el área de educación primaria
}

\author{
Curriculum Integration as a Learning Experience: A Roadmap for Its Implementation in Two \\ Teacher Training Courses in the Area of Primary Education
}

\section{Integração curricular como experiência de aprendizagem: Um roteiro para sua aplicação em dois cursos de formação de professores na área do ensino fundamental}

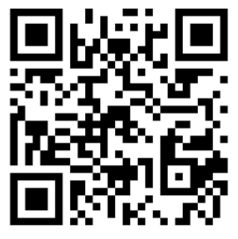

Resumen:

Introducción. Este artículo sistematiza la experiencia de integración curricular, efectuada por las profesoras del curso DBJ 402 Didáctica de las Ciencias y las Matemáticas para la Educación Básica y DBJ 403 Desarrollo de la Niñez en I y II Ciclo, de la carrera de Pedagogía con énfasis en I y ll Ciclo de la Educación General Básica de la División de Educación Básica (DEB), en el año 2018. La organización del proceso, su implementación, la valoración por parte de las docentes y la percepción estudiantil se desarrolla en este documento. Objetivo. Enriquecer la formación del estudiantado y fortalecer los objetivos y temáticas de ambos cursos. Metodología. Se explican las fases con las diferentes acciones que se efectuaron para poner en práctica la integración curricular; implementando tres estrategias que favorecieron la vinculación entre saberes y el aprendizaje, tanto para las académicas como para el grupo estudiantil. Como parte del proceso de sistematización de la experiencia se aplicó un cuestionario para recabar la percepción de las personas estudiantes acerca de la experiencia, así como determinar las fortalezas y las recomendaciones, expresadas por el estudiantado, con respecto a las estrategias llevadas a cabo. Análisis de resultados. Ejecutar esta práctica integradora requirió 
http://doi.org/10.15359/ree.25-3.19

http://www.una.ac.cr/educare

educare@una.ac.cr

compromiso, planificación y una nueva mirada del quehacer docente por parte del profesorado universitario, además de la participación activa de sus estudiantes. Conclusión. Implementar estas prácticas representa un reto para el profesorado universitario.

Palabras claves: Articulación; currículo; estudiantado universitario; formación docente; integración curricular; rol docente.

\begin{abstract}
:
Introduction. This article systematizes the experience of curricular integration, carried out in 2018 by the teachers of DBJ 402 Didactics of Sciences and Mathematics for Basic Education and DBJ 403 Child Development in I and II Cycle courses, of the Pedagogy career with an emphasis in I and II Cycle of the Basic General Education of the Basic Education Division (DEB). The process organization, its implementation, the teachers' assessment, and the student perception are developed in this paper. Objective. To enrich student training and strengthen the objectives and topics of both courses. Method. The phases with the different actions carried out to implement the curricular integration are explained, implementing three strategies that favored the link between knowledge and learning, both for the teachers and the student group. As part of the systematization process of the experience, a questionnaire was applied to gather the students' perceptions about the experience and determine the strengths and recommendations expressed by the students regarding the carried-out strategies. Analysis of results. Implementing this integrative practice required commitment, planning, and a new look at the teaching work by the university faculty, besides the active participation of students. Conclusions. Implementing these practices represents a challenge for university teachers.
\end{abstract}

Keywords: Articulation; curriculum; university students; teacher training; curriculum integration; teaching role.

\title{
Resumo:
}

Introdução. Este artigo sistematiza a experiência de integração curricular, realizada pelos docentes do curso DBJ 402 Didática das Ciências e Matemática para o Ensino Básico e DBJ 403 Desenvolvimento da Infância nos Ciclos I e ll, da carreira de Pedagogia com ênfase em I e ll Ciclo da Educação Básica Geral da Divisão de Educação Básica (DEB), no ano de 2018. A organização do processo, sua implementação, a avaliação pelos professores e a percepção dos estudantes são desenvolvidas neste documento. Objetivo. Enriquecer a formação dos estudantes e fortalecer os objetivos e temas de ambos os cursos. Metodologia. São explicadas as fases com as diferentes ações realizadas, para colocar em prática a integração curricular; implementar três estratégias que favoreceram o vínculo entre conhecimento e aprendizado, tanto para acadêmicos quanto para o grupo de estudantes. Como parte do processo de sistematização da experiência, foi aplicado um questionário para saber a percepção dos estudantes sobre a experiência, bem como determinar os pontos fortes e as recomendações que foram expressas por eles sobre as estratégias adotadas. Análise de resultados. A execução dessa prática integrativa exigiu comprometimento, planejamento e uma nova visão do ensino por parte do corpo docente da universidade, além da participação ativa dos estudantes. Conclusão. A implementação dessas práticas representa um desafio para os professores universitários.

Palavras-chaves: Articulação; currículo; estudantes universitários; formação de professores; integração curricular; papel da pessoa docente. 
http://doi.org/10.15359/ree.25-3.19

http://www.una.ac.cr/educare educare@una.ac.cr

\section{Introducción}

La Comisión de Gestión Académica y Curricular del Centro de Investigación y Docencia en Educación (CIDE), de la Universidad Nacional de Costa Rica, emitió el oficio CIDE-VD-281-2010 (comunicación personal), en el que establece tres áreas de abordaje de su modelo pedagógico: integración curricular, incorporación de tecnologías e investigación.

Al respecto, la División de Educación Básica, Unidad Académica que pertenece al CIDE y que tiene a su cargo la formación docente de las carreras de Pedagogía, escogió el área de integración curricular, que determina el diseño y desarrollo de las tareas docentes como los procesos de aprendizaje que se promueven en el estudiantado universitario, (Zabalza, 2012), mediante acciones coordinadas por nivel.

A partir de lo anterior, las docentes de los cursos DBJ 402 Didáctica de las Ciencias y las Matemáticas para la Educación Básica y DBJ 403 Desarrollo de la Niñez en I y II Ciclo, de la carrera de Pedagogía con énfasis en I y II Ciclo de la Educación General Básica de la DEB decidieron, en el II ciclo lectivo del 2018, llevar a cabo un proceso de integración curricular entre los cursos, que pretendía generar una propuesta pedagógica integradora, innovadora y pertinente dentro de la formación docente, al realizar acciones conjuntas, mediante la articulación de temáticas y prácticas pedagógicas que conjugan saberes de las materias.

Este proceso de integración curricular requirió de la planificación mediante una hoja de ruta con una serie de fases para su consecución y se definieron tres estrategias de integración, con el fin de fortalecer los objetivos y temáticas de ambos cursos. El objetivo del presente artículo es compartir esta hoja de ruta implementada en la experiencia de integración curricular, para el enriquecimiento de la formación profesional de los futuros grupos docentes.

\section{Contextualización teórica}

La integración curricular refiere a la forma de organizar los contenidos formativos en un plan de estudios; en este caso particular en una carrera universitaria, para lograr una mayor articulación, coherencia y coordinación entre los cursos involucrados en la acción formativa que se lleva a cabo.

Al respecto, Costa y del Río (2016), para referirse al tema, establecen el término articulación horizontal entendido como:

Los esfuerzos que deben realizarse entre distintas asignaturas que se cursan en forma simultánea para lograr un diálogo que facilite a los alumnos el tránsito por las mismas, a partir del uso de un lenguaje común, que permita reconocer en las distintas asignaturas los objetos comunes que aparecen en formatos diferentes y una metodología coherente que permita unificar "reglas de juego". (p. 150) 
http://doi.org/10.15359/ree.25-3.19

http://www.una.ac.cr/educare

educare@una.ac.cr

La integración curricular requiere el aporte de todos los campos profesionales involucrados, como señalan Alonso et al. (2011), esta sustituye la individualidad que define el trabajo docente, por un trabajo cooperativo, permitiendo articular las diferentes materias.

Señalan Rousserie et al. (2012, p. 341) que "es preciso dar coherencia y unidad al sistema de contenidos, entendiéndose a este como el trabajo articulado entre las diferentes áreas del conocimiento que intervienen en el proceso de enseñanza-aprendizaje".

El currículo integrado, al establecer nexos entre los diferentes cursos del plan de estudios, supera la visión fragmentada y desarticulada que ha prevalecido tradicionalmente, evidenciando las vinculaciones que existen entre ellos, para un aprendizaje más significativo y estructurado para el estudiantado. Al respecto, Chaves Álvarez et al. (2011) agregan que la integración les ofrece una experiencia de aprendizaje más enriquecedora, dado que permite reflexionar sobre las problemáticas de forma integrada. Además, promueven, según Tabash Blanco (2015), la criticidad y la creatividad al integrar los conocimientos.

En este sentido, Zabalza (2012) indica que la implementación de acciones curriculares integradas conlleva innovar el currículo, lo que significa que el personal docente conciba y piense su quehacer de manera diferente y cambie su concepción de lo que entiende por enseñanza y aprendizaje.

La integración curricular considera el currículo como proyecto formativo integrado. Zabalza (2012) ofrece una explicación de cada uno de esos elementos; como proyecto la integración se presenta como propuesta clara y con visión de conjunto, superando la perspectiva de considerarla como una sumatoria de partes. El sentido formativo es entendido como "un conjunto de mejoras y aprendizajes en los sujetos que irían desde el dominio de las asignaturas que cursan (no todas ellas necesariamente profesionalizantes) hasta la adquisición de competencias genéricas que los enriquezcan para la vida" (Zabalza, 2012, p. 22), que contribuye en el área profesional, pero también en la personal; en otras palabras, un futuro profesional capaz de desenvolverse como sujetos "críticos, autónomos, competentes capaces de construir su propia identidad en relación con los demás y su entorno" (Rousserie et al., 2012, p. 341). Finalmente, el aspecto de la integralidad supera la visión del currículo como conjunto de materias sin ninguna relación entre sí y en la que la integralidad provee de coherencia a las materias.

Al concebir el currículo como proyecto formativo integral hace de la integración curricular un proceso innovador que establece los planes de estudios como sistemas y favorece las estrategias y estructuras "que permitan garantizar la coherencia y continuidad del proyecto formativo" (Zabalza, 2012, p. 26). 
http://doi.org/10.15359/ree.25-3.19

De esta forma, el currículo integrado va a incidir no solo en la manera en que se diseñan, organizan e implementan las tareas propias del profesorado, sino además en los procesos de aprendizaje que se desarrollan con el estudiantado.

Señala Rousserie et al., (2012) que este proceso no se logra rápidamente, sino que se caracteriza por requerir más tiempo, para desarrollarse poco a poco. Ante lo cual expone una seriede estrategias que permiten llevar a cabo el proceso de forma paulatina, como lo es el establecimiento de espacios comunes a varias materias, conceptualizada como espacios compartidos entre diferentes cursos en los que el estudiantado hace algún trabajo o práctica y ponen en ejercicio lo aprendido en las diferentes materias articuladas. De acuerdo con esta descripción, la experiencia de integración curricular presentada en este artículo se puede enmarcar en este tipo de dinámica, porque se integraron espacios y se converge con otras disciplinas.

\section{Cursos vinculados en la propuesta}

Los cursos que desarrollaron la propuesta de integración fueron el curso DBJ 402 Didáctica de las Matemáticas y las Ciencias para la Educación Básica y el curso DBJ 403 Desarrollo de la Niñez en I y II Ciclo.

\section{Curso Didáctica de las Matemáticas y las Ciencias para la Educación Básica}

Como objetivo general del curso se planteó “Reflexionar sobre la mediación pedagógica de las principales áreas que conforman las ciencias naturales y las matemáticas en el I y ll Ciclos de la Educación General Básica, aplicando una visión de integralidad" (Universidad Nacional, División de Educación Básica, 2018a, p. 1).

\section{Curso Desarrollo de la Niñez para I y II Ciclo}

Como objetivos del curso se plantearon "Reflexionar sobre los diferentes conceptos y particularidades del desarrollo humano, para un replanteamiento de su práctica educativa", así como "Elaborar propuestas educativas para la estimulación del desarrollo humano integral" (UNA, DEB, 2018b, p. 2). En la metodología del curso se implementó el taller "como un espacio de comunicación pedagógica en el que permanentemente se construyen y reconstruyen categorías teóricas, significados y discursos" (Hernández, 2009, p.72).

\section{Marco metodológico}

Para la consecución del objetivo propuesto se establecieron dos líneas de trabajo: la construcción de la hoja de ruta con el plan de acción para la integración curricular y las fases que muestran la secuencia detallada del proceso. 
http://doi.org/10.15359/ree.25-3.19

http://www.una.ac.cr/educare

educare@una.ac.cr

\section{Participantes}

Participaron en la propuesta 32 estudiantes de la carrera de Pedagogía con énfasis en I y II Ciclo de la Educación General Básica, de tercer año de la carrera, matriculados en los dos cursos que se integraron.

\section{Instrumento}

Como parte del proceso de sistematización de la experiencia, se aplicó un cuestionario de preguntas abiertas, para evaluar el proceso y conocer las percepciones del estudiantado participante, sobre cada una de las estrategias de integración implementadas, valorar fortalezas de estas y las recomendaciones. El instrumento incluyó una pregunta general sobre la implementación de los trabajos integrados, así como consultas sobre fortalezas y recomendaciones hacia cada una de las tres estrategias de integración realizadas.

El cuestionario, como instrumento que conjunta una serie de preguntas, es según Hernández Sampieri et al. (2010), uno de los instrumentos más utilizados para recolectar datos $y$, en este caso, se planteó esa serie de consultas como preguntas abiertas.

Las percepciones indicadas por el estudiantado fueron sistematizadas en una matriz, mediante la siguiente categorización: enriquecimiento en la formación, visión integradora, estrategia efectiva para el aprendizaje, planificación en los trabajos integrados, estrategia de integración sobre el taller de sexualidad, estrategia de integración sobre el taller lúdico y estrategia de integración sobre la propuesta metodológica.

\section{Mapeo del proceso}

\section{Primera línea de trabajo: Construcción de la hoja de ruta}

Esta línea de trabajo implicó la planificación de la hoja de ruta como un proceso continuo de generación de acciones y conocimientos dirigidos a lograr los fines propuestos. Se planificaron tres estrategias de integración curricular como parte de los dos cursos durante el II ciclo lectivo de 2018, se estableció el abordaje de las acciones pedagógicas a ejecutar, considerando los objetivos de los cursos. Se seleccionaron espacios, recursos materiales y humanos requeridos. La Figura 1 muestra la hoja de ruta elaborada para la propuesta de integración, visualizando las líneas de acción, las fases, el tiempo y el objetivo. 
http://doi.org/10.15359/ree.25-3.19

http://www.una.ac.cr/educare educare@una.ac.cr

Figura 1: Hoja de ruta de la propuesta de integración curricular

\section{La integración curricular como experiencia de aprendizaje}

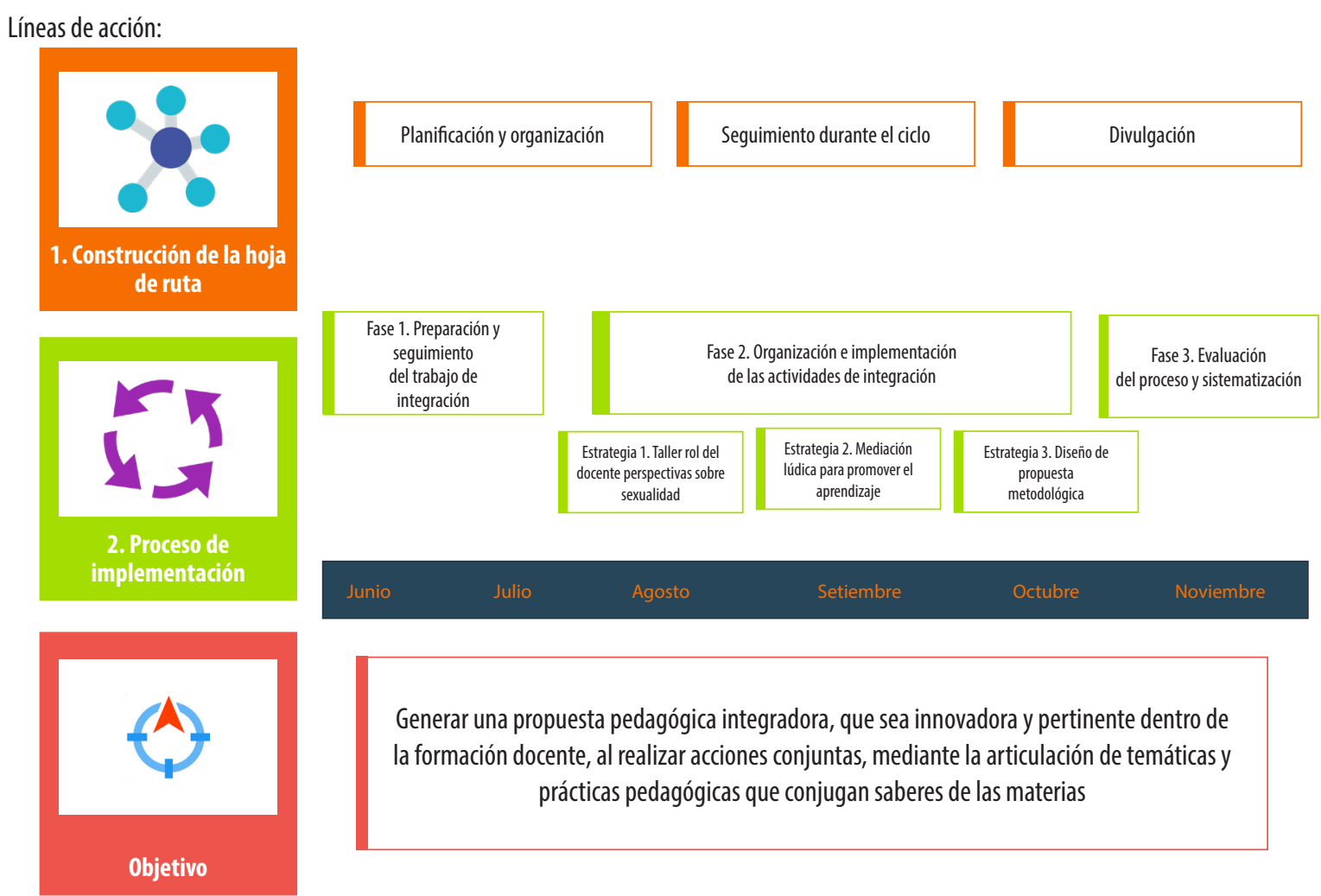

Nota: Elaboración propia.

\section{Segunda línea de trabajo: Desarrollo del proceso realizado para la implementación de la hoja de ruta}

Para el logro de la integración curricular, como experiencia de aprendizaje, con la hoja de ruta se plantearon una serie de fases, que permitieron al profesorado y al estudiantado comprender cada una de las acciones, su propósito, temática, características y valoración.

\section{Fase 1. Preparación y seguimiento del trabajo de integración curricular}

En esta fase las profesoras organizaron la propuesta de integración académica entre los dos cursos, se definió el objetivo, las acciones a realizar en reuniones previas al inicio de las clases y se dio seguimiento al proceso antes de cada actividad. Se mantuvo una comunicación constante vía correo electrónico y mensajería para finiquitar detalles de las actividades y plantear los instrumentos de evaluación como parte de la estrategia evaluativa de cada curso. 
http://doi.org/10.15359/ree.25-3.19

http://www.una.ac.cr/educare

educare@una.ac.cr

\section{Fase 2. Organización e implementación de las actividades de integración}

\section{Se establecieron tres estrategias de integración, detalladas a continuación:}

\section{Estrategia de integración 1. Taller sobre el rol del docente: Perspectivas sobre sexualidad}

Esta estrategia se realizó en conjunto, contó con una especialista en psicología que desarrolló un taller sobre el tema de sexualidad, generando un espacio de fundamentación teórica y de reflexión. Posteriormente, se asignó la elaboración de un planeamiento, enfocado en alguno de los temas del taller y del Programa de Ciencias del MEP. Esta tarea se trabajó en la sesión posterior de clases y la integración temática se evaluó desde el curso de Desarrollo de la Niñez.

\section{Estrategia de integración 2. Mediación lúdica para promover el aprendizaje}

Esta estrategia se realizó en un espacio abierto, con la participación de un especialista en recreación, abordando la relevancia del juego como estrategia de aprendizaje en el desarrollo de la niñez en el ámbito escolar y su implementación para el abordaje de los contenidos de las asignaturas básicas. Se consideraron los conocimientos previos, se implementaron dinámicas colaborativas y competitivas, y posteriormente con las profesoras, se realizó el análisis y resolución de una guía de trabajo para reforzar conceptos vinculados con el juego, la recreación y la incorporación de las estrategias lúdicas en la labor docente.

\section{Estrategia de integración 3. Diseño de propuesta metodológica}

Consistió en la construcción de una propuesta metodológica, se planificaron, de forma integrada, contenidos del Programa de estudio de Matemática y Ciencias del MEP, y se consideraron, a su vez, las áreas del desarrollo humano integral. Este, para ser aplicado en un grupo de escolares de primero a sexto grado. Durante el diseño se explicó al estudiantado la plantilla de planeamiento, la cual incluía un espacio para la identificación de las áreas del desarrollo abordadas con las diferentes estrategias metodológicas propuestas en el planeamiento integrado de las asignaturas de Ciencias y Matemáticas. La especificidad de las áreas del desarrollo se relacionó con lo abordado desde el curso Desarrollo de la Niñez y los contenidos y estrategias correspondieron al curso de Didáctica de las Ciencias y las Matemáticas.

\section{Fase 3. Evaluación del proceso y sistematización}

La evaluación del proceso se realizó al finalizar las estrategias de integración, mediante el cuestionario aplicado. Para la sistematización de la información se formularon categorías, que permitieron ordenar las percepciones y analizar la lógica del proceso desarrollado e identificar los factores positivos de las estrategias y los elementos a reforzar. La sistematización, 
http://doi.org/10.15359/ree.25-3.19

por tanto, según Gorina Sánchez y Alfonso Berenguer (2013), "es interpretada como un rasgo del procesamiento de la información que resulta de hacer una integración sistemática de las partes esenciales de la información, de sus propiedades y relaciones, de sus aspectos generales y singulares, de su unidad y multiplicidad, que hacen síntesis en un todo concreto" (p. 48).

De la primera pregunta del cuestionario sobre la implementación de los trabajos integrados, surgieron categorías relacionadas con el enriquecimiento en la formación docente, la aplicación de visión integradora, la integración como estrategia efectiva para el aprendizaje y la importancia de la planificación en los trabajos integrados.

Del taller del rol docente y las perspectivas sobre sexualidad se extrajeron fortalezas como la calidad de la información, la persona facilitadora, el enriquecimiento de los conocimientos, y se recomendaron aspectos relacionados con dinamismo, ampliación de tiempo y ampliación de las temáticas. En el taller lúdico para el aprendizaje surgieron fortalezas como la actividad novedosa y eficaz para el quehacer docente y se recomendó la distribución diferente del tiempo, la implementación de actividades para todos, el cuidado de la seguridad de las personas participantes y el complementar con otros recursos. Para el diseño de la propuesta metodológica se plantearon categorías relacionadas con la promoción del aprendizaje, la inclusión del desarrollo integral en la planificación, la promoción del trabajo en equipo y el disfrute. Por su parte, las recomendaciones se relacionaron con ajustes en la implementación del planeamiento y el acompañamiento durante el proceso.

\section{Interpretación crítica}

La propuesta de integración curricular implementada, al ser un proceso de innovación que enriquece la calidad de la formación docente, como lo indica Zabalza (2012), determina tanto el diseño y desarrollo de las tareas docentes, como los procesos de aprendizaje que se promueven en el estudiantado universitario, promoviendo significatividad en lo aprendido.

Superar la organización curricular tradicional por una nueva perspectiva de currículo integrado resultó un reto para el equipo docente; significó una labor de organización y negociación conjunta; desarrollar reuniones antes, durante y al final de la implementación del proceso; negociar y coordinar las diferentes acciones articulando cronogramas, especialistas invitados, espacios, trámites, dinámicas y recursos; acordar estrategias de evaluación conjuntas para que el estudiantado visualice otro elemento integrador; ceder espacios para la construcción conjunta del aprendizaje, esfuerzos que enriquecen las temáticas y objetivos de ambos cursos; y la aplicación más vivencial del Modelo Pedagógico de la UNA, que indica que el profesorado debe "organizar situaciones de enseñanza vinculadas a las áreas de conocimiento que interrelacionen y articulen la teoría y la práctica, ... con propósitos y fundamentos que permiten la reflexión y la conformación de conocimientos más sistemáticos" (UNA, 2007, p. 8). 
http://doi.org/10.15359/ree.25-3.19

http://www.una.ac.cr/educare

educare@una.ac.cr

De acuerdo con lo expuesto, para lograr un proceso articulado el personal docente debe comprometerse, mostrar flexibilidad, apertura al diálogo y al trabajo colaborativo, para mirar de forma diferente los procesos formativos y asumir que sus acciones pueden lograr transformarlos.

En los procesos de integración curricular, el estudiantado desempeña un rol esencial, en el sentido de que es protagonista y beneficiario de los objetivos que se esperan lograr. Por tanto, el rol del profesorado se complementa con la participación y compromiso de cada estudiante, que se involucra en las experiencias como sujeto de aprendizaje, como constructor de los saberes conceptuales, procedimentales y actitudinales, así como evaluador del proceso de integración.

\section{Análisis de resultados}

De las principales percepciones del estudiantado -identificados para efectos de confidencialidad con la letra $\mathrm{P}$ (participante) y diferenciados cada uno con una letra minúsculase destacaron aspectos muy valiosos que refuerzan la necesidad de seguir ejecutando estas propuestas. Entre estos elementos sobresalen los siguientes:

\section{Enriquecimiento en la formación}

El estudiantado indicó que los trabajos de integración son muy relevantes, ya que permiten valorar diferentes áreas en las que se encuentra el alumno (Pb); se aprovecha el tiempo, se trabajan temas desde diferentes puntos de vista (Pk), así como ayuda al crecimiento personal y académico para el estudiantado y el cuerpo docente (Pñ); cuando se trabaja en grupo se fortalece la amistad con el otro y mayor interacción (PI). Además, si el profesorado toma conciencia sobre este tipo de propuestas integradas, se logrará que los trabajos den buen resultado y sobre este caso en particular opinaron que existió una buena integración y que las actividades fueron exitosas y ayudaron al crecimiento del estudiante universitario (Pi).

Destacaron también en este aspecto, que la integración les permitió visualizarse en su rol como futuras personas docentes, sea implementando la integración con sus propio estudiantado o en el trabajo con otros miembros del cuerpo docente, esto al señalar que cuando seamos docentes vamos a tener una idea más clara de la integración (Po); además reconocieron que cuando estemos en el aula tendremos que trabajar con otros docentes, ... es un ejemplo de lo que debemos hacer en la escuela (Pq); al unificar los planeamientos, estamos ayudándonos a nosotros y a los chicos a la hora de poder interactuar varias materias en un mismo tema (Pv).

Relacionado también con la formación, señalaron la necesidad de que más cursos se integren para que se aproveche más el aprendizaje y el tiempo. Indicaron que nos evita muchos trabajos que son repetitivos y dejándonos mucho más aprendizajes en ambos cursos a la vez, por lo que es de suma importancia integrar conocimientos (Ps); la carga académica para los estudiantes es menor (Pk); nos ayuda a aprovechar de una mejor manera el tiempo, a la vez que liviana [sic] el estrés y la presión, que al mismo tiempo para los docentes es mucha presión ( $\mathrm{PW}$ ). 
http://doi.org/10.15359/ree.25-3.19

Es claro que el estudiantado respalda la realización de experiencias integradoras como un elemento que enriquece su formación docente, tanto durante la etapa formativa como para su futura labor docente. La integración propuesta permite el diálogo de saberes, vinculados no solo a los cursos en particular, sino en la profundización de su preparación profesional y en su quehacer docente en general.

\section{Visión integradora}

El estudiantado opinó que se logró una visión integradora pues se ayudó a crear una visión integrada sobre los distintos aspectos relacionados con el área profesional (Pd), integrar materias que tal vez no se conocía se podían integrar ( $\mathrm{Pa})$; ver el cómo se pueden integrar diferentes materias y además son más enfocadas a tener un aprendizaje significativo (Pe); relacionar contenidos de una materia a otra (Pf). Además, indicaron que les pareció pertinente la propuesta, pues es una iniciativa muy buena y efectiva ya que nos formamos de una manera integral, buscamos relaciones entre todos los cursos y a la vez contribuye a que se nos facilita el trabajo (Pj); ya que permite disminuir la carga académica (Pa).

Agregaron que esta experiencia les dio otra visión, los trabajos integrados brindan una perspectiva un poco más amplia de los procesos de enseñanza, así como una integración de factores educativos que actualmente se requieren $(\mathrm{Pu})$. Se reconoció el valor del compromiso del profesorado, se evidencia que existe buena relación entre docentes y se dan los contenidos de una forma más integral como se supone que se quiere hacer en el aula (Px).

La propuesta permitió demostrar al estudiantado, de forma vivencial, cómo lograr una mediación con una visión integradora de los aprendizajes, a través de diversas estrategias, que permitan una formación integral del futuro personal docente.

\section{Estrategia efectiva para el aprendizaje}

Según las percepciones del estudiantado, reconocieron que la propuesta es una estrategia efectiva, interesante y útil para promover el aprendizaje, pues no solo se requiere de articular el proceder docente, sino la integración de contenidos, ya que al final, se debe aplicar todo como un conjunto de habilidades y no por separado, por esto estas estrategias son apropiadas (Pm) y con esta estrategia el proceso de formación se da unificado y no segmentado (Pr).

A su vez, señalaron que la estrategia de integración es un reto y un proceso de innovación, lo que la hace oportuna para sus propósitos. Indicaron que se enriquecieron las sesiones de trabajo grupal, además fue todo un reto (Pc) y es una estrategia innovadora y creativa, los estudiantes aprenden de manera conjunta, haciendo lo dado en el aprendizaje significativo (Pp). 
http://doi.org/10.15359/ree.25-3.19

http://www.una.ac.cr/educare

educare@una.ac.cr

La integración curricular se visualiza como una estrategia para lograr mayor efectividad en el aprendizaje y es considerada como un proceso innovador, lo cual debería ser una práctica cotidiana en la formación docente, dado el reto curricular de superar la segmentación de los aprendizajes.

\section{Planificación en los trabajos integrados}

Como fortaleza, el estudiantado opinó sobre la relevancia de la planificación para lograr estrategias exitosas. Reconocieron en la propuesta la claridad en lo proyectado y la necesaria planificación, elemento que se ha destacado en este artículo, como fundamental para la organización y negociación de estos procesos.

Señalaron que los trabajos implementados fueron muy acertados (Pg); excelentes, siempre y cuando haya una buena organización (Pn); sumamente provechosos, siempre y cuando estos tengan un fin y objetivo claro, que permita aprender ligando de dos formas correctas (Ph); y resaltaron la planificación como un aspecto vinculado con la significatividad, ya que se necesita una buena planeación para que se vea como un aprendizaje significativo (Pz).

La elaboración previa de una hoja de ruta para implementar la experiencia de integración curricular ofrece claridad en el propósito y la realización de las estrategias de mediación, así como un seguimiento oportuno del aprendizaje logrado por el estudiantado y una valoración constante de la labor del profesorado que la lleva a cabo. Se genera, por tanto, un enriquecimiento de la formación profesional de los futuros cuerpos docentes y resulta en una herramienta dinámica que puede ser implementada en otras experiencias similares de integración curricular.

Estos cuatro elementos planteados como fortalezas de la propuesta de integración refuerzan la perspectiva de que este tipo de trabajos, si bien requiere compromiso, tiempo y organización entre docentes, enriquece su formación y permite al estudiantado fortalecer sus propios conocimientos, articulando saberes, así como servirle de modelaje para su futuro ejercicio de la profesión.

Por otra parte, es valioso reconocer, desde la perspectiva estudiantil, cuáles pueden ser las recomendaciones para que este tipo de propuestas sean aún mejores. A ese respecto, se hace una reflexión sobre las tres estrategias de integración implementadas, en las cuales el estudiantado señaló algunos aspectos que se profundizan a continuación.

\section{Estrategia de integración sobre el taller de sexualidad}

A partir de la valoración del taller implementado, se sistematizaron las opiniones del estudiantado con respecto a sus fortalezas y recomendaciones. Señalan como aspectos positivos la calidad de la información, el desempeño del facilitador y el enriquecimiento de los conocimientos aportados al estudiantado. Entre los aspectos de mejora se definieron 
http://doi.org/10.15359/ree.25-3.19

el dinamismo, la ampliación de la temática a otros contextos, la aplicación no forzada y la ampliación de las temáticas abordadas.

El estudiantado, con respecto a la calidad de la información, indicó que estos conocimientos reforzaron sus saberes con respecto al tema del desarrollo sexual o les ofrecieron nuevos conocimientos, un mayor aprendizaje y sobre todo poder aclarar dudas sobre ciertos temas (Pv); además, se visualizó la importancia de la didáctica, pues señalaron que la información fue de gran relevancia en cuanto al aprendizaje y también la dinámica de cómo manejar temas así (Pñ).

Un elemento similar se identificó en la fortaleza relacionada con el enriquecimiento de los conocimientos, el estudiantado logró identificar articulación de ambos cursos, dado que mencionó que el taller le proporcionó conocimiento sobre la aplicación en el aula (Pa), así como el logro de un mayor conocimiento, ya que nos ayudan a tener más claro las etapas de las y los alumnos (Pb).

Con respecto al desempeño del facilitador, el estudiantado resaltó su preparación y conocimiento de la temática, la expositora estaba muy bien preparada y tuvo el calor y carisma necesarios para interactuar con nosotros (Pj); además los aspectos relacionados con el rol docente, quien debe ser un sujeto profesional conocedor de los contenidos a impartir, así como con variedad de estrategias para mediar el aprendizaje. Mostró gran manejo del tema y gran agilidad para explicar los diferentes contenidos, uso del juego didáctico lo que permite vivenciar estrategias para aplicarlas en el futuro (Ph).

Sin embargo, se destacó como recomendación que se planteen en el taller actividades más lúdicas, con un mayor dinamismo en la actividad, pues reconocen que el juego es esencial y una herramienta necesaria para el aprendizaje de escolares.

También se recalcó la necesidad de ampliación de las temáticas sobre sexualidad, pues son saberes que como futuros grupos docentes desean reforzar. Se comprendió que como es un taller, el tiempo fue limitado, por lo que recomendaron una ampliación de temáticas a otros contextos, que sea más un curso que un taller o que se facilite este tipo de talleres en los centros educativos. Finalmente, agregaron que la integración debe ser un proceso de aplicación no forzada, pues se puede desviar la intención propuesta y no lograr los objetivos planteados.

En general, se logró una comprensión de la conexión que existe entre ambos cursos, sus temáticas y contenidos, además que el grupo de estudiantes logró visualizar cómo es la integración curricular y cómo puede ser llevada a cabo o utilizada en su quehacer docente.

\section{Estrategia de integración sobre el taller lúdico}

Se sistematizaron las percepciones del taller lúdico según la valoración del estudiantado con respecto a sus fortalezas y recomendaciones. Con respecto a sus fortalezas señalaron que generó aspectos positivos en el estudiantado en dos sentidos principalmente. El primero, al 
http://doi.org/10.15359/ree.25-3.19

http://www.una.ac.cr/educare

educare@una.ac.cr

recalcar que dentro de su formación esta actividad lúdica fue novedosa, diferente e interactiva, pues les permitió relacionarse más como grupo y ser una actividad pertinente a sus edades, adecuada a su propio desarrollo como jóvenes, dinámica diferente a lo común, excelente para relacionarlo con lo que se debe hacer en el aula (Pa), Genial y dinámico, muy acorde a nuestras edades y a la carrera (Pz). El otro elemento fue el ser una actividad eficaz para su quehacer docente, pues Me amplió mucho el pensamiento de cómo integrar lo lúdico en las clases (Po), se reconoce la relevancia que tiene el juego como estrategia para promover el aprendizaje y les permitió entender que es posible la integración del juego con los contenidos de las diferentes materias.

Con respecto a las recomendaciones, el estudiantado acotó aspectos relacionados con la distribución del tiempo, para una mejor organización de las actividades y que se permitiera profundizar más, para lograr un mejor aprovechamiento del proceso. Se valoró la relevancia de la planificación y la organización previa de los eventos, sus respuestas así lo evidenciaron, al indicar Un poco más de preparación previa con respecto a las actividades para profundizar mejor $(\mathrm{Pa})$, además que se debió considerar la implementación de actividades para todos, es decir, que fueran atinentes a todas las personas del grupo, Tomar en cuenta las necesidades de todos los estudiantes (Pw). Otra recomendación fue el cuidado de la seguridad de las personas participantes, contar con una planificación no solo permite organizar las estrategias, sino también evitar o atender posibles contratiempos, lo que el estudiantado planteó como velar por la seguridad de los participantes $(\mathrm{Pz})$, pues no se puede estar exento de contrariedades cuando se trabaja con grupos de personas y dinámicas lúdicas.

Otra recomendación muy valiosa, es que haya un complemento de la experiencia con otros recursos, que permita la documentación de las acciones y la oportunidad de crear nuevas actividades lúdicas con el grupo de compañeros y compañeras, intercambiar opiniones y estrategias; a la vez que sería importante brindar más opciones para comentar o vivenciar dinámicas que puedan desarrollarse, no solo en espacios más abiertos, sino también a nivel de aula o en espacios reducidos, dadas las diferentes condiciones que se pueden encontrar en las instituciones educativas.

\section{Estrategia de integración sobre la propuesta metodológica}

Las percepciones del estudiantado sobre el diseño de la propuesta metodológica permiten identificar fortalezas relevantes a considerar como la visualización de la integración curricular, la inclusión del desarrollo integral en la planificación, la promoción del trabajo en equipo y el disfrute. A su vez, las sugerencias para la propuesta metodológica se relacionan con los ajustes que se requirieron en la ejecución del planeamiento, así como el acompañamiento durante el proceso.

En las respuestas dadas por el estudiantado se logró la visualización de la integración curricular y comprender cómo se ven las vinculaciones entre los temas, comprender que se puede integrardos asignaturas al mismo tiempo, además abarcar de manera más divertida cada asignatura $(\mathrm{PI})$, además la estrategia utilizada permitió al estudiantado realizar un análisis integral de la 
http://doi.org/10.15359/ree.25-3.19

tarea solicitada desde los cursos. Un aspecto fundamental es considerar las áreas del desarrollo en el planeamiento y el poder justificar las acciones pedagógicas asumidas como docentes según la inclusión del desarrollo integral en la planificación: Excelente, me encantó, aprendimos a dar una justificación personal a nuestras actividades en el aula, basadas en desarrollo, genial (Pr).

Este tipo de propuesta metodológica permitió el trabajo colaborativo y la promoción del trabajo en equipo, aspecto que promovió el intercambio de ideas y percepciones, un reto más para la construcción del aprendizaje. Además, el estudiantado señaló que en este tipo de experiencia se alcanza el disfrute, indistintamente si les fue fácil la integración o si se les complicó, lo que se relaciona con su comprensión de lo que implica la integración, así como el reforzamiento obtenido en las dos primeras estrategias implementadas por las profesoras.

Además, el estudiantado indicó dos sugerencias a considerar, una referida a los ajustes que se requirieron en la ejecución del planeamiento, pues al ser una experiencia, recomendaron hacer más este tipo de planeamientos, así como el identificar un centro educativo para su ejecución, pues en esta oportunidad no se logró implementar propiamente el planeamiento con escolares, para vivenciar las reacciones de estudiantes menores y conocer los resultados de una aplicación integrada, dada la situación de huelga que afectó al país en el sector educativo. La otra recomendación planteada giró en torno al acompañamiento durante el proceso, pues, si bien se proporcionaron las directrices y ejemplificaciones por parte de las profesoras, el estudiantado solicitó más apoyo mayor explicación de los aspectos puntuados a tratar, para lograr ejecutar el trabajo de forma más clara (Ph) , considerando que estas experiencias de integralidad son nuevas para eel grupo y un reto como parte de su formación, lo que no contradijo su percepción de que son propuestas enriquecedoras para el aprendizaje.

En general, se puede indicar que esta última estrategia de integración le permitió al estudiantado concientizar lo aprendido para la interiorización de saberes. El diseño de la propuesta metodológica promovió una práctica consciente y culminar el proceso de integración propuesto por las profesoras de los cursos. Particularmente, lograron la comprensión sobre la integración curricular de dos asignaturas (Matemática y Ciencias), en relación con el abordaje de las áreas del desarrollo; aspectos eficazmente articulados en el diseño de la propuesta metodológica.

\section{Conclusiones y recomendaciones}

Generar una propuesta pedagógica integradora, que sea innovadora y pertinente dentro de la formación docente, demanda para su implementación acciones conjuntas de articulación de temáticas y de prácticas pedagógicas que permitan conjugar los saberes de los cursos involucrados en este tipo de procesos.

Esto comprende un reto para el profesorado universitario, le demanda, más allá de realizar las acciones pedagógicas, el romper esquemas, pensar de otra manera sobre su quehacer 
http://doi.org/10.15359/ree.25-3.19

http://www.una.ac.cr/educare

educare@una.ac.cr

docente y realizar una apuesta diferente en el estilo de trabajo. Le exige una organización diferente; un cambio en la forma tradicional de implementar su curso; un trabajo colaborativo con sus compañeros o compañeras docentes; una negociación proactiva de espacios, estrategias metodológicas y evaluativas. Así como el concientizarse de su nuevo rol, de proyectarse en un trabajo con otras personas para el fortalecimiento de la formación docente del estudiantado, de un nivel de compromiso mayor hacia el éxito de su curso y el de los cursos del resto del profesorado; asumiendo el currículo de forma integrada y no como la suma de partes.

De esta manera, se logrará una incidencia mayor en el currículo como tal y en el perfil del estudiantado, pues la vivencia de alternativas de integración desde su formación le dará a la persona estudiante perspectivas claras sobre que el aprendizaje es uno solo y que es más significativo cuando se visualiza de forma integrada. Estas iniciativas superan el espacio de acción del personal docente que las ejecuta, por lo que se requiere una implementación de todo el colectivo docente que forma parte de la carrera.

Las experiencias de integración curricular se pueden realizar de forma pertinente si existe la disposición por parte del profesorado de asumir ese reto como responsable de la mediación de los cursos universitarios. Además, es fundamental la anuencia al diálogo y a la negociación propositiva, al ser parte del engranaje de las acciones a desarrollar en equipo. Se hace palpable que el implementar estrategias de integración va a requerir esfuerzos organizativos y atcadémicos antes, durante y después de cada estrategia; por ello, el valor de la planificación detallada de las acciones y el reconocimiento del rol del profesorado y del estudiantado en esas mismas es indispensable. De allí, la relevancia de contar con una hoja de ruta para implementar la experiencia de integración curricular, que permita el enriquecimiento de la formación profesional de los futuros docentes y el seguimiento por parte de las personas a cargo de los cursos que se integran al proceso. Así mismo, la elaboración de esta hoja de ruta se convierte en una herramienta que puede ser implementada en otras experiencias similares de integración curricular.

Además, las experiencias de integración curricular enriquecen la propia visión del estudiantado con respecto a su proyección profesional, pues le permiten al docente en formación ser consciente que como en su futuro como docente también deben integrar saberes y promover acciones compartidas de aprendizaje con sus estudiantes. Esto se logra cuando, desde la formación, se vivencian este tipo de propuestas de forma organizada y procesual, de manera que el estudiantado pueda ir comprendiendo en qué consiste la integración curricular que se le propone desde el hacer y no solo desde la teoría.

Se concluye que, tras la propuesta implementada por las profesoras, el estudiantado logró percibir, conceptualizar y comprender en qué consiste la integración curricular. En las percepciones del estudiantado se puede observar el impacto que tuvieron las diferentes estrategias, pues es factible observar en sus opiniones cómo logran analizar y conceptualizar 
http://doi.org/10.15359/ree.25-3.19

http://www.una.ac.cr/educare educare@una.ac.cr

el proceso, vincular los contenidos y las estrategias, las cuales consideran que pueden ser utilizadas también en diferentes cursos y no únicamente en un nivel de la carrera o en solo unas asignaturas a cargo de profesorado interesado en articular esfuerzos.

De esta manera, se logra además la formación de profesionales en educación con más compromiso con su propio proceso de aprendizaje; así como se promueve la interacción y construcción de aprendizajes más significativos. Esto, por cuanto en las acciones que se propongan para articular, el estudiantado deberá ser parte esencial del proceso, un actor activo del aprendizaje que se desea lograr y un evaluador de las acciones, lo cual conlleva a enriquecer su formación y a reconocer la importancia de un aprendizaje significativo al construir saberes mediante acciones innovadoras.

Además, al lograr el estudiantado hacer conexiones entre lo aprendido y su utilidad en su futuro como docente, está transfiriendo ese aprendizaje a su cotidianidad y a futuras situaciones pedagógicas, lo cual le permitirá reforzar su perfil profesional, valorar la interacción y la integración como propuestas eficaces para la discusión y enriquecimiento de conocimientos, así como para fomentar la innovación y la utilización de diversas estrategias y recursos en su mediación pedagógica actual y futura.

\section{Declaración de conflictos}

No existe ninguna clase de conflicto en la implementación del proceso de integración curricular y el desarrollo de este artículo.

\section{Referencias}

Alonso, M. J. Arandia, M., Esnaola, M., Hornilla, J. M., Novella, I., Pastor, D., Ruiz de Gauna, M. P. y González, V. (2011). El proceso de integración curricular: Articulando el conocimiento académico y profesional. Revista de Educación Social, (13), 1-11. https://www.eduso.net/ res/pdf/13/proce res_13.pdf

Chaves Álvarez, A. L., Hernández Vargas, D., León Sánchez, J., Pereira Pérez, Z. y Vargas Dengo, M. C. (2011). La integración curricular: Una experiencia en el primer nivel de diplomado de la carrera de Licenciatura en Pedagogía con énfasis en Educación Preescolar. Revista Electrónica Educare, 15(2), 63-86. https://doi.org/10.15359/ree.15-2.6

Costa, V. A. y del Río, L. S. (2016). La articulación en la enseñanza. En C. Giordano y G. Morandi (Presidencia), 1 a Jornadas sobre las prácticas docentes en la Universidad Pública: Transformaciones actuales y desafíos para los procesos de formación. Universidad Nacional de La Plata. http://sedici.unlp.edu.ar/bitstream/handle/10915/61283/Documento completo.pdf-PDFA.pdf?sequence=1\&isAllowed=y 
http://doi.org/10.15359/ree.25-3.19

http://www.una.ac.cr/educare

educare@una.ac.cr

Gorina Sánchez, A. y Alfonso Berenguer, I. (2013). Modelo de la dinámica formativa del procesamiento de la información en las investigaciones sociales. Didasc@lia: Didáctica y Educación, 4(1), 31-56. https://dialnet.unirioja.es/servlet/articulo?codigo=4233583

Hernández, A. M. (2009). El taller como dispositivo de formación y de socialización de las prácticas. En L. Sanjurjo (Coord.), Los dispositivos para la formación en las prácticas profesionales (pp.71-104). Homo Sapiens Ediciones. https://pdfcoffee.com/los-dispositivos-para-laformacion-en-las-practicas-profesionales-sanjurjo-2-pdf-free.html

Hernández Sampieri, R., Fernández Collador, C. y Baptista Lucio, P. (2010). Metodología de la investigación. McGrawHill.

Rousserie, H. F., Maydana, A., Cardozo, S., Inchauspe, M. y Merro, P. (Setiembre, 2012). La articulación de contenidos como facilitador en la enseñanza de las ciencias agrarias. En C. Miceli (Presidencia). IV Congreso Nacional y III Congreso Internacional de Enseñanza de las Ciencias Agropecuarias, Universidad Nacional de La Plata. http://sedici.unlp.edu.ar/ bitstream/handle/10915/21634/Documento completo.pdf?sequence=1

Tabash Blanco, N. (2015). La integración curricular y el uso del libro de texto en la escuela primaria. Revista de Lenguas Modernas, (22), 391-404. https://revistas.ucr.ac.cr/index.php/ rlm/article/view/19696/19775

Universidad Nacional. (2007). Modelo pedagógico. Autor. http://www.documentos.una.ac.cr/ bitstream/handle/unadocs/9724/Modelo\%20Pedagogico.pdf?sequence=1\&isAllowed=y

Universidad Nacional, División de Educación Básica (2018a). Programa de curso DBJ 402 Didáctica de las Ciencias y las Matemáticas para la Educación Básica. Autor.

Universidad Nacional, División de Educación Básica. (2018b). Programa de curso DBJ 403 Desarrollo de la Niñez en I y ll Ciclo. Autor.

Zabalza, M. A. (2012). Articulación y rediseño curricular: El eterno desafío institucional. Revista de Docencia Universitaria, 10(3), 17-48. https://doi.org/10.4995/redu.2012.6013 